\title{
Vaccinating All will Save All-The World Needs to Hurry up and Unite against COVID-19
}

\author{
Abhijit V Boratne $^{1} \odot$ Amrit Mishra $^{2}$ (D) \\ Keywords: COVID-19, G7 summit, India, Pandemic, Vaccination drive, Vaccine, World Health Organization. \\ SBV Journal of Basic, Clinical and Applied Health Science (2021): 10.5005/jp-journals-10082-03121
}

Dear Editor,

At the press briefing on COVID-19 in June 2021, the WHO Director General commented on the present scenario of the world's vaccination rates. He stated that the nations with the highest vaccination rates have been considering lifting off the lockdown restrictions. With the increased global transmission of COVID-19 virus variants, lifting restrictions too quickly could be disastrous for those who are not vaccinated yet.' Currently, as of July 8, 2021, the COVID-19 cases have soared to reach 184.8 million globally and 30.7 million in India. Also, only a little more than 3 billion vaccine doses have been administered worldwide. ${ }^{2}$

But then, not all countries have been successful in vaccinating their public, even with the first dose. On the one hand, the highincome countries have accounted for administering almost $44 \%$ of the world's doses. On the other hand, the low-income countries have barely met $0.4 \%$. This is a matter of concern because this percentage has not improved much since the vaccines were first rolled out. The WHO Director General called for a massive global effort to vaccinate at least $10 \%$ of the population of all countries by September, and at least 30\% by the end of the year. ${ }^{1}$

When the cases of the first wave of the pandemic began to plummet, many nations were relieved and unfortunately let their guard down. It was presumed that the pandemic was almost over. Factors like disregarding the warnings of experts, inadequacy in stockpiling medical supplies, enhancing infrastructure and augmenting healthcare workforce, lag of momentum in research, and vaccine complacency were evident in catalyzing the second wave of the pandemic to go out of control. ${ }^{3}$

On May 1, 2021, the fourth phase of the COVID-19 vaccination drive opened up to the entire adult population in India. But the number of people getting jabbed since the start of the vaccination drive was quite low. The average number of daily vaccine jabs administered was just 16 lakh per day in the first week of May. As of July 5,2021 , India has managed to vaccinate only $27 \%$ of its total population with at least one dose. ${ }^{4}$

But just focusing on improving one country's vaccination statistics is not central in wading off the pandemic. India is a giant vaccine manufacturer, accounting for $60 \%$ of vaccine supply to the world through Serum Institute of India, Pune. But at present, it is struggling to meet even its own country's demand. ${ }^{5}$ At the G7 summit held during June 11-13, 2021, in the United Kingdom, the world leaders have promised to donate 100 crore COVID-19 vaccine doses to poorer nations, either directly or through the earlier COVAX scheme (with collaboration of the World Health Organization, the Global Alliance for Vaccines and Immunizations and the Coalition for Epidemic Preparedness Innovations). ${ }^{6}$ India being a democratic country can meet the global vaccination demand with proper

\footnotetext{
1,2Department of Community Medicine, Mahatma Gandhi Medical College and Research Institute, Puducherry, India

Corresponding Author: Amrit Mishra, Department of Community Medicine, Mahatma Gandhi Medical College and Research Institute, Puducherry, India, Phone: +91 9487738287, e-mail: amritmishra92@ gmail.com

How to cite this article: Boratne AV, Mishra A. Vaccinating All will Save All-The World Needs to Hurry up and Unite against COVID-19. J Basic Clin Appl Health Sci 2021;4(3):77-78.

Source of support: Nil

Conflict of interest: None
}

infrastructure and resources. Hence, all developed nations must make haste and come forward to assist India by providing funds, information, expertise, and raw materials.

The world is one big family divided by borders. All the people are equally responsible for the health and well-being of their family. Only when every person gets vaccinated, we as a family will be able to avert this danger.

\section{The Eight Strategies to Improve Vaccination in India}

- The Government can collaborate with both management and social institutions and medical experts for sorting out material planning, funding, and management. This can sort out the logistics and enhance the rate of COVID-19 vaccine production and distribution

- Facilitation to expedite availability, accessibility, and utilization of all available human resources in healthcare system to administer rolled-out COVID-19 vaccines, along with potential upcoming candidates namely Zydus Cadila, Johnson \& Johnson, etc.

- With many COVID-19 vaccines available in near future, the urban healthcare facilities will be flooded with vaccine recipients. In order to reduce this burden, we need to utilize all the available rural healthcare facilities (e.g., primary health centers, community health centers, and subcenters) to accommodate the vaccination demand and to avoid movement of rural population to urban areas.

- The Government should keep an eye on the probability of overpricing.

- Incentivizing and/or announcement of prospective withdrawal of the benefits of government schemes, if people do not get vaccinated.

- To organize vaccination camps in villages, tribal, hilly, and remote areas with a view to strengthen the periphery. 
- To decentralize the vaccination centers and ensure that vaccine supplies are provided to organizations (both government and private) to cover all their employees within a stipulated timeline.

- To involve all registered non-governmental and voluntary organizations of India to educate and mobilize the vaccinationeligible population within their field practice areas.

\section{OrCID}

Abhijit V Boratne ㄴ https://orcid.org/0000-0001-7698-303X

Amrit Mishra ㄴ) https://orcid.org/0000-0003-0830-3084

\section{References}

1. Hindustan Times. Lifting Covid-19 restrictions too quickly can be disastrous for those not vaccinated:WHO Chief. 2021. Available from: https://www.hindustantimes.com/world-news/lifting-covid-19- restrictions-too-quickly-can-be-disastrous-for-those-not-vaccinatedwho-chief-101623092761664.html.

2. World Health Organization. WHO Coronavirus (COVID-19) dashboard. 2021. Available from: https://covid19.who.int.

3. Krishnan A, Dasgupta R. Science, policy, people, and public health: What Is COVID-19 teaching us? Indian J Public Health 2020;64 (Suppl. S2):S87-S89. DOI: 10.4103/ijph.IJPH_540_20.

4. Hindustan Times. India has administered over 357 million Covid vaccine doses till now. 2021. Available from: https://www. hindustantimes.com/india-news/india-has-administered-over357-million-covid-vaccine-doses-till-now-101625547973749.html.

5. McGregor G. The world's largest vaccine maker can't keep up with the world's worst COVID wave. 2021. Available from: https://fortune. com/2021/04/20/india-covid-cases-vaccine-vaccinations-seruminstitute.

6. Lee J, Morton B. G7: World leaders promise one billion Covid vaccine doses for poorer nations. 2021. Available from: https://www.bbc.com/ news/uk-57461640. 